\title{
Glomerular Size and Structure in Diabetes Mellitus
}

\author{
III. Early Enlargement of the Capillary Surface \\ J.P. Kroustrup, H. J. G. Gundersen, and R. Østerby \\ The University Institute of Pathology, and the Second University Clinic of Internal Medicine, Arhus, Denmark
}

Summary. The present electron microscopic study shows that the kidney hyperfunction in early diabetes can be due to a significant morphological change: an increased glomerular filtration surface. Applying standard stereological methods, the area of the peripheral wall of the glomerular capillaries was measured in biopsy specimens obtained from 7 patients with early diabetes and 7 controls. - An 80 per cent enlargement of the capillary wall (the surface of the peripheral basement membrane) was found in the diabetics $(2 p=0.0096)$. Also the total area of the interface between the tuft and the urinary space was increased by 70 per cent $(2 \mathrm{p}=$ $0.029)$. Since the thickness of the peripheral basement membrane is known to be unchanged in patients with early diabetes the finding of an increased area of the membrane implies that an increased quantity of basement membrane material is present in these patients. The significance of this phenomenon for the understanding of the metabolism of the basement membrane is discussed, and a working hypothesis is advanced for the pathogenesis of the diabetic microangiopathy.

Key words: Early diabetes, glomerular hypertrophy, capillary surface, filtration surface, basement membrane, diabetic microangiopathy, kidney hyperfunction.

It is well-known that the glomerular filtration rate (GFR) is elevated in juvenile diabetics when determined shortly after the onset of the disease, and that the elevation of the GFR persists at least during the first decade [3]. The light microscopic demonstration of an enlargement of the glomerular tuft and of the capillary luminal volume [10] in early diabetes indicates that morphological changes accompany the increased GFR. The mechanism leading to the increase in capillary luminal volume could be either an increased luminal pressure resulting in an unfolding of the capillary wall, or an enlargement of the capillary wall area.

To distinguish between these two alternative possibilities, each of which could theoretically be responsible for the increased GFR, it is necessary to determine directly the area of the capillary wall. This is not possible at the light microscopic level, but it can be done on electron micrographs.

\section{Material and Methods}

Kidney biopsy material was available from seven newly diagnosed juvenile diabetics, who were biopsied several weeks to a few months after the acute onset of the disease and again after 5 (3 to 7) weeks of insulin treatment. Six of these patients (case Nos. 8 to 13) are described in a previous paper [10], which also includes case No. 15 , with a duration of diabetes of one year. The control group of young subjects without clinical signs of diabetes or renal disease is identical to that in the same study, except that one subject (a 20 year old female) is omitted from the present series since no material was available for electron microscopy. The specimens were fixed in $\mathrm{OsO}_{4}$, embedded in Vestopal $\mathrm{W}$, and thin sections were cut from randomly selected glomeruli. From each biopsy three complete glomerular crosssections (in one case only one) were photographed with a Philips EM 200 at low magnification. The exact magnification of each film was calculated on photographs of a diffraction grating replica with 28800 lines per inch and ranged from 3666 to $3893 \times$.

\section{Stereological methods}

Each glomerular tuft was circumscribed by the minimal convex polygon. The area of the polygon is denoted the 'tuft area' and is used as the reference area.

On the prints the three interfaces between the capillary lumina, the mesangial regions, and the uri- 


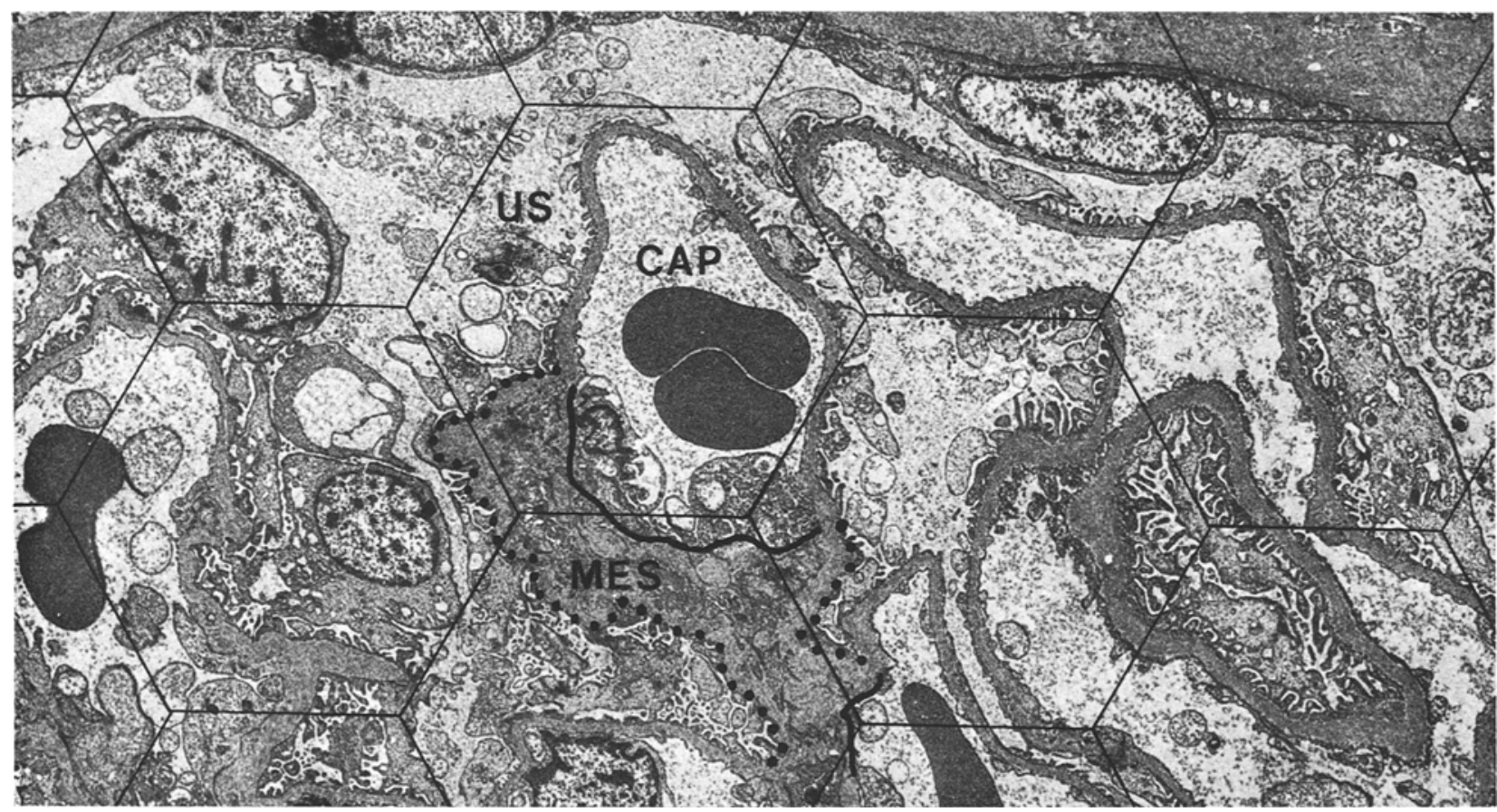

Fig. 1. Part of a glomerular tuft with the employed grid shown at the magnification of 3666 . The hexagon has a side length of $26 \mathrm{~mm}$, corresponding to $7.1 \mu$. The grid consequently has a point density of 0.015 points $/ \mu^{2}$ and a line density of $0.023 \mu / \mu^{2}$. CAP: capillary lumen, US: urinary space, MES: mesangial region. The limits between mesangial region and capillary lumen (full-drawn line) and between mesangial region and urinary space (dotted line) are indicated

nary space were defined as illustrated in Figure 1. Capillary lumen/urinary space (CAP/US): the luminal margin of the peripheral basement membrane; Mesangial region/capillary lumen (MES/CAP): the luminal margin of the mesangial region; Mesangial region/urinary space (MES/US): the urinary margin of the mesangial region.

The surface densities of these interfaces were estimated by standard stereological methods [2]. If $\mathrm{C}$ denotes the total number of intercepts between an interface and a randomly thrown array of test-lines with a total length of $\mathrm{L} \mu$, then

$\mathrm{S}_{\mathrm{V}}=2 \frac{\mathrm{C}}{\mathrm{L}} \mu^{-1}$

$S_{V}$ is the surface density of the interface, i. e. the area per unit volume of the glomerular tuft.

Repeated determinations of $\mathrm{S}_{\mathrm{V}}$ from the same prints of three whole glomerular cross sections showed a coefficient of variation of 7.9 per cent.

The individual mean glomerular volume (V), corrected to a body surface of $1.73 \mathrm{~m}^{2}$, was known from the above-mentioned light microscopic study [10]. The absolute surface (S) of an interface per mean glomerulus could then be calculated:

$$
\mathrm{S}=\mathrm{V} \times \mathrm{S}_{\mathrm{V}} \mu^{2}
$$

On the assumption that the number of glomeruli per individual is invariant, differences in S between individuals is proportional to differences in the total area of the surface per individual.

In order to determine whether an unfolding of the capillary wall is present, the surface/volume ratio of the capillary was measured. The stereological determination of this ratio [1] is carried out in analogy with that of $S_{V}$, but the reference area is that of the capillary lumen instead of the glomerular tuft. Other things being equal, an unfolding of the capillary wall means that an increased capillary volume is enclosed within an unchanged wall area, i.e. a fall in the surface/volume ratio of the capillary is a measure of the degree of unfolding.

All counting procedures were performed by the same investigator who was unaware of the identity of the specimens. The measurements were carried out over an average total tuft area of $54000 \mu^{2}$ per biopsy.

Since distributions of absolute areas generally are lognormal the comparisons between groups have been carried out on logarithmically transformed values. The group mean $( \pm S D)$ calculated from these distributions equals the geometric mean of the original values multiplied by/divided by $(X / \div)$ the tolerance factor.

Student's t-test was employed in the statistical treatment, using a 5 per cent limit of significance.

\section{Results}

The surface densities of the three interfaces in controls and diabetics are given in Table 1. Comparison of the biopsies obtained from the six diabetics before and after treatment revealed no consistent trends in any of the estimated surface densities. 
Consequently, means of the estimates before and after treatment are given in Table 1. The surface density of the interface between the capillary lumen and the urinary space (CAP/US) was increased in diabetics compared to controls: $0.187 \pm 0.018 \mu^{-1}$ versus $0.169 \pm 0.014 \mu^{-1}$ (mean $\pm S D$ ). The difference just fell short of statistical significance $(2 \mathrm{p}=$ 0.051 ). There were no differences between diabetics and controls in the surface densities of the mesangial surfaces towards the capillary and towards the urinary space (Table 1 ).

The calculated absolute areas per glomerulus of the three interfaces are shown in Figure 2. The area of the peripheral capillary wall of $0.244 \times 1 \div 1.52$ $\mathrm{M}^{2}$ (geometric mean and tolerance factor) in diabetics is 80 per cent larger than that of the controls: $0.136 \times 1 \div 1.32 \mathrm{Mu}^{2}$. The $2 \mathrm{p}$ value of this difference between the absolute areas is 0.0096 . Concerning the absolute areas of the mesangial surfaces, comparison of the MES/CAP and the MES/US interfaces in controls and diabetics revealed no statistically significant differences, the values being $0.065 \times 1 \div 1.44 \mathrm{M} \mu^{2}$ versus $0.099 \times / \div 1.67$ $\mathrm{Mu}^{2}(2 \mathrm{p}=0.10)$ and $0.067 \times / \div 1.51 \mathrm{Mu}^{2}$ versus $0.097 \times / \div 1.85 \mathrm{M}^{2}(2 \mathrm{p}=0.21)$, respectively.

The area of the total tuft surface towards the urinary space (CAP/US + MES/US) was also calculated in each individual. The mean value of this surface was $0.342 \times 1 \div 1.58 \mathrm{M} \mu^{2}$ in diabetics and $0.204 \times / \div \mathrm{M} \mu^{2}$ in controls. The difference of 70 per cent is statistically significant $(2 \mathrm{p}=0.029)$.

The measure of folding of the capillary wall, i. e. the capillary surface/volume, was $0.81 \pm 0.14 \mu^{-1}$ (mean $\pm \mathrm{SD}$ ) in the diabetics and $0.76 \pm 0.13 \mu^{-1}$ in the controls $(2 \mathrm{p}=0.51)$.

\section{Discussion}

The demonstrated increase in the absolute surface area of the peripheral capillary wall and of the total tuft/urinary interface in glomeruli from patients with early diabetes could well be the morphological correlate of the elevation of GFR in such patients. In groups of diabetics similar to the patients in the present study estimates of the increase in GFR range from 41 to 26 per cent, before and after treatment, respectively [3]. A direct comparison between these figures and the estimated increase in the absolute surface area of the capillary wall of 80 per cent has meaning only on the assumption that all other factors affecting GFR (differential hydrostatic and colloid osmotic pressure, hydraulic conductivity, etc.) are unchanged. Nothing is known about the validity of such assumptions at present.

The increased absolute volume of the glomeruli in diabetics demonstrated earlier [10] and the unchanged or elevated surface densities of the three interfaces measured separately in this study imply that the corresponding absolute surfaces are in-
Table 1. Surface densities $\left(\mu^{-1}\right)$ of the interfaces between capillary lumen and urinary space (CAP/US), mesangial region and capillary lumen (MES/CAP), and mesangial region and urinary space (MES/US) in controls and in patients with early, juvenile diabetes. In the diabetics means of the estimates before and after treatment are given

\begin{tabular}{|c|c|c|c|}
\hline $\begin{array}{l}\text { Subject } \\
\mathrm{nr} .\end{array}$ & Cap/U.S. & Mes/Cap & Mes/U.S. \\
\hline \multicolumn{4}{|l|}{ Controls } \\
\hline 1 & 0.182 & 0.082 & 0.082 \\
\hline 2 & 0.180 & 0.062 & 0.061 \\
\hline 3 & 0.146 & 0.079 & 0.080 \\
\hline 4 & 0.183 & 0.075 & 0.103 \\
\hline 5 & 0.170 & 0.093 & 0.077 \\
\hline 6 & 0.159 & 0.090 & 0.084 \\
\hline 7 & 0.162 & 0.089 & 0.101 \\
\hline mean & 0.169 & 0.081 & 0.084 \\
\hline $\mathrm{SD}$ & 0.014 & 0.011 & 0.014 \\
\hline \multicolumn{4}{|l|}{ Diabetics } \\
\hline 8 & 0.207 & 0.066 & 0.043 \\
\hline 9 & 0.195 & 0.091 & 0.094 \\
\hline 10 & 0.176 & 0.075 & 0.076 \\
\hline 11 & 0.179 & 0.078 & 0.088 \\
\hline 12 & 0.156 & 0.078 & 0.078 \\
\hline 13 & 0.176 & 0.076 & 0.082 \\
\hline 14 & 0.205 & 0.078 & 0.084 \\
\hline 15 & 0.201 & 0.069 & 0.070 \\
\hline mean & 0.187 & 0.076 & 0.077 \\
\hline $\mathrm{SD}$ & 0.018 & 0.007 & 0.016 \\
\hline difference & 0.018 & -0.005 & -0.007 \\
\hline SEM & 0.008 & 0.005 & 0.008 \\
\hline $2 \mathrm{p}$ & 0.051 & 0.30 & 0.37 \\
\hline
\end{tabular}

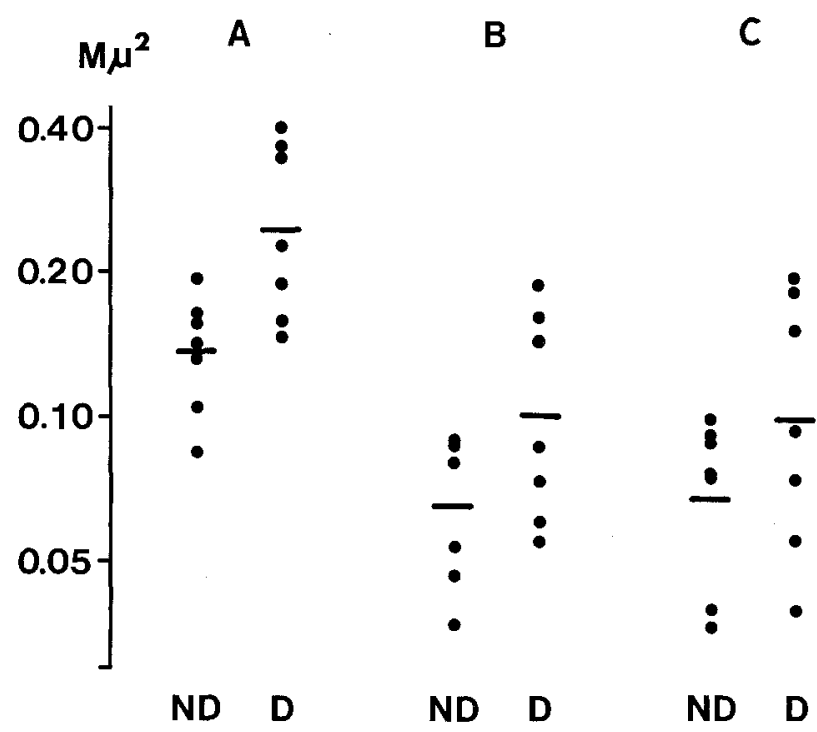

Fig. 2. The absolute surface area $\left(\mathrm{M}^{2}\right)$ per mean glomerulus of the interfaces between capillary lumen and urinary space (A), mesangial region and capillary lumen (B), and mesangial region and urinary space (C) in controls (ND) and patients with juvenile diabetes (D). All areas are normalized to a body surface of 1.73 $\mathrm{m}^{2}$. Since no material was available for the light-microscopical determination of the absolute glomerular volume, the areas in patient no. 14 (Table 1) are missing. The ordinate is logarithmic. Horizontal bars indicate mean values 
creased. Although this increase reached statistical significance only concerning the peripheral capillary wall, the area of the two mesangial surfaces showed a tendency in the same direction. Due to the scatter and the limited number of observations the probability of a statistical type II error is quite high. Larger groups are therefore needed before definite conclusions can be drawn.

Since unfolding of the capillary wall would lead to a decreased surface/volume ratio of the glomerular capillaries, the finding of an unchanged ratio in patients with early diabetes excludes the possibility that unfolding is the mechanism behind the increase of the capillary luminal volume demonstrated by light microscopy [10].

Since evidence of an increased filtration surface in diabetics has been absent until now, several authors have proposed an increased filtration pressure as the mechanism behind the increased GFR. However, the increased filtration pressure was deduced from the measured increase in filtration fraction, and the finding of an enlarged filtration surface in the present study invalidates this deduction.

The observed morphological changes also have implications concerning the metabolism of the basement membrane in diabetes. The increase in the absolute area of the peripheral basement membrane, together with the fact that the thickness of the same basement membrane is unchanged [9], indicate that glomeruli in early diabetics contain increased quantities of basement membrane, presumably due to an increased rate of synthesis. This would fit with the observation that the activity in the renal cortex of an enzyme involved in basement membrane synthesis is increased shortly after the induction of experimental diabetes in rats and is normalized after insulin treatment [8].

Other results from animal studies give further support to the assumption that the enlargement of the renal corpuscles is brought about by the metabolic derangement. An increase in the size of the whole kidney, as well as in glomerular size, has been demonstrated in rats within days or weeks of the induction of streptozotocin-diabetes $[5,6$, Seyer-Hansen, personal communication]. Furthermore, after 7 days of diabetes, the kidney size in rats is closely correlated with the elevation in the blood glucose concentration [7].

In diabetic patients firm evidence has been obtained that the elevated GFR [3] and the increased kidney size [4] are both restored to practically normal values after strict insulin treatment for several weeks. After 3 to 7 weeks of insulin treatment no consistent change was observed in the glomerular structures in the present series, but after this short period only a pronounced reversal would be detectable in such a small group. No further data are available on the issue of reversibility of the glomeru- lar enlargement by strict diabetic control, but at present it is only reasonable to assume that in the glomeruli structural changes parallel those of the functional parameters.

We now propose, as a working hypothesis, that enlargement of the glomerular capillary wall, i. e. increased synthesis of the peripheral basement membrane triggered by the metabolic aberrations characterizing diabetes mellitus, constitutes the earliest phase in the development of diabetic microangiopathy. After a short time growth of the capillary surface is stopped by mechanisms counteracting the glomerular hyperfunction. The persistently high rate of synthesis then results in an increasing thickness of the basement membrane, eventually leading to the glomerulosclerosis of long-term diabetes. This unitary hypothesis implies that information about diabetic microangiopathy can be obtained from experiments of much shorter duration than previously necessary.

Acknowledgements. We are very much indebted to Drs. G. Gregersen, B. Møller, V. Posborg and H. Skjoldborg for the kidney biopsy material. We wish to thank Mrs. Kirsten Gerlach for skilful assistance. The study was supported by grants from Nordisk Insulin Fond and Statens lægevidenskabelige Forskningsråd.

\section{References}

1. Chalkley, H.W., Cornfield, J., Park, H.: A method for estimating volume-surface ratios. Science 110, 295-297 (1949)

2. Elias, H., Hennig, A., Schwartz, D.E.: Stereology: Applications to biomedical research. Physiol. Rev. 51, 158-200 (1971)

3. Mogensen, C. E.: Kidney function and glomerular permeability to macromolecules in juvenile diabetes. Dan. Med. Buil. Suppl. 3 (1972) (Thesis)

4. Mogensen, C.E., Andersen, M.J.F.: Increased kidney size and glomerular filtration rate in early juvenile diabetes. Diabetes 22, 706-712 (1973)

5. Ross, J., Goldman, J.K.: Effect of streptozotocin-induced diabetes on kidney weight and compensatory hypertrophy in the rat. Endocrinology 88, 1079-1082 (1971)

6. Seyer-Hansen, K.: Renal hypertrophy in streptozotocindiabetic rats. Clin. Sci. 51, 551-555 (1976)

7. Seyer-Hansen, K.: Renal hypertrophy in experimental diabetes: Relation to severity of diabetes. Diabetologia 13, 141-143 (1977)

8. Spiro, R. G., Spiro, M. J.: Effect of diabetes on the biosynthesis of the renal glomerular basement membrane: Studies on the glucosyltransferase. Diabetes 20, 641-648 (1971)

9. Østerby, R.: Early phases in the development of diabetic glomerulopathy. A quantitative electron microscopic study. Acta Med. Scand. Suppl. 574 (1975) (Thesis)

10. Østerby, R., Gundersen, H. J. G.: Glomerular size and structure in diabetes mellitus. I. Early abnormalities. Diabetologia 11, 225-229 (1975)

Received: October 28, 1976, and in revised form: January 4, 1977

Dr. Ruth Østerby

Patologisk Anatomisk Institut

Kommunehospitalet

DK-8000 Århus C, Denmark 